Acta Cryst. (2002). A58 (Supplement), C301

STRUCTURE AND FUNCTION ANALYSIS OF A FAMILY OF CYSTEINE-RICH HYPOTHETICAL PROTEINS FROM THE HUMAN PATHOGEN HELICOBACTER PYLORI

Peer R.E. Mittl Lucas Luethy Markus G. Gruetter University of Zurich Biochemical Institut Winterthurer Strasse 190 ZURICH 8057 SWITZERLAND

The genome sequence of the human pathogen Helicobacter pylori contains a family of cysteine-rich hypothetical proteins that so far lack a functional and three-dimensional description. Four members of this family, designated Helicobacter cysteine-rich proteins (Hcp), have been expressed recombinatly in $E$. coli, refolded and purified. It was shown that the proteins HcpA, -B, -C and -D are capable of binding and hydrolysing $\beta$-lactam antibiotics. The substrate profiles characterize these proteins as penicillinases rather than cephalosporinases. The structural analogy between $\beta$-lactams and D-Ala,DAla peptides suggests an implication of Hcp proteins in cell-wall biosyntesis. $\mathrm{HcpB}$ and $-\mathrm{C}$ were crystallized and the structures were refined at $1.95 \AA$ and $2.50 \AA$ resolution, respectively. The structures reveal modular architectures of $\alpha / \alpha$-repeats that are distantly related to the tetratricopeptide-repeats. The HcpB structure also shows a bound ligand that was copurified with the protein. The electron density suggests that this ligand might be a $\mathrm{N}$-acetyl-muramic acid (NAM), a ubiquitous compound of the bacterial cell-wall, that was validated by mass-spectrometry.

Keywords: CYSTEINE-RICH, $\beta$-LACTAMASE, HYPOTHETICAL PROTEIN
Acta Cryst. (2002). A58 (Supplement), C301

AUTOMATION OF MACROMOLECULAR DATA COLLECTION INTEGRATION OF DATA COLLECTION AND DATA PROCESSING $\underline{\text { H. Powell }}^{1}$ A.G.W. Leslie ${ }^{1}$ G. Winter ${ }^{1}$ C. Nave ${ }^{2}$ E.M.H. Duke ${ }^{2}$ S.H. Kinder ${ }^{2}$ D. Love $^{2}$ S. McSweeney ${ }^{3}$ O. Svensson ${ }^{3}$ D. Spruce ${ }^{3}$ S. Delageniere ${ }^{3}$

${ }^{1}$ Mrc-Lmb MRC Center Hills Road CAMBRIDGE CB2 2QH UK ${ }^{2}$ Daresbury Laboratory ${ }^{3}$ European Synchrotron Radiation Facility

The data collection programs at the SRS Daresbury (PXGEN++) and the ESRF Grenoble (ProDC) have been close coupled to Mosflm via TCP/IP sockets through an expert system and a linking server program to provide a high level of automation. A characterize crystal button on the data collection GUI initiates the automatic determination of appropriate data collection parameters for single crystals of previously unknown space group. The characterize crystal command collects two images from a crystal (at $0^{\circ}$ and $90^{\circ}$ in phi), and then sends an instruction to the Mosflm server which is forwarded to Mosflm to conduct the characterization. Mosflm proceeds to (i) autoindex each image individually and also both together, (ii) estimate the effective mosaicity, (iii) integrate the first image to determine the effective resolution limit, and (iv) calculate a suitable data collection strategy to give maximum completeness for both unique and anomalous data. An expert system developed jointly at SRS and ESRF then makes further decisions regarding data collection based on these results. The Mosflm server has been developed to provide an extendable interface to a next-generation GUI, and also gives simple access to the program for other interfaces. Improvements to Mosflm itself allow the appropriate sequence of operations to be carried out in a flexible and robust manner.

Keywords: AUTOMATED DATA COLLECTION DATA PROCESSING

Acta Cryst. (2002). A58 (Supplement), C301

\section{A NEURAL NET APPROACH TO THE AUTOMATED ANALYSIS OF CRYSTALLIZATION TRIALS}

G. Spraggon $^{1}$ A. T. Kreusch ${ }^{1}$ S. A. Lesley ${ }^{1}$ J. P. Priestle ${ }^{2}$

${ }^{1}$ Genomics Institute of The Novartis Research Foundation Protein Structure and Funcation 10675 John Jay Hopkins Drive SAN DIEGO 92912 USA

${ }^{2}$ Novartis Pharma AG, Basel, Switzerland

An important tool for the Structural Genomics initiative will be a means to automatically detect crystals in crystallization experiments, as well as classifying other non-crystallizing trials. Such information will not only relieve the many man hours of tedious visual inspection but may also provide directions to proceed in subsequent crystallization experiments and a metric for the design of better crystallization screens. A computer program, Crystallization Experiment Evaluation Program (CEEP), has been developed which carries out this procedure classifying crystallization experiments into 5 broad categories (invalid experiment, clear drop, homogenous precipitant, inhomogeneous precipitant, crystal hit). The program consists of two main parts, a preprocessor for determining the drop boundary and extracting features from within the drop and a classification engine in the form of a selforganizing Kohonen neural net. The algorithm has currently been tested on over 500,000 images derived from crystallization experiments performed with the entire proteome of Thermotoga maritima. Crystallization experiments were robotically generated in a submicrolitre, sitting drop vapour diffusion, 96 well format with 3 images taken over a period of 28 days for each individual experiment. The results and problems of the analysis are presented using various classification vectors as well as with the incorporation of time series information.

Keywords: STRUCTURAL GENOMICS, CRYSTALLIZATION, IMAGE ANALYSIS crystallization system.

LLNL is operated by UC for US DOE under contract W-7405-ENG-48. Work was funded by NIH-P50-GM62410 center grant. 